\title{
Tendencias en la normatividad, el desarrollo tecnológico y la aplicación de motores eléctricos de alta eficiencia
}

\author{
Trends in standardization, technology development \\ and applications of energy efficient motors
}

\author{
Javier A. Rosero-García ${ }^{1}$, Enrique C. Quispe ${ }^{2}$, Rosaura del Pilar Castrillón-Mendoza ${ }^{3}$ \\ ${ }^{1}$ Dr.-Ing. Departamento de Ingeniería Eléctrica y Electrónica, Universidad Nacional de Colombia, EMED, Bogotá-Colombia \\ ${ }^{2}$ Dr.-Ing. Departamento de Energética y Mecánica, Universidad Autónoma de Occidente, GIEN, Cali-Colombia \\ ${ }^{3}$ M.Sc. Departamento de Energética y Mecánica, Universidad Autónoma de Occidente, GIEN, Cali-Colombia \\ E-mail:ecquispe@uao.edu.co
}

Recibido: 26/09/17

Aceptado: 11/01/18
Cite this article as: J. Rosero-Garcia, E.C. Quispe, R. Castrillon-Mendoza, “Trends in standardization, technology development and applications of energy efficient motors", Prospectiva, Vol 16, N 1, 83-90, 2018.

\section{RESUMEN}

La crisis energética y ambiental ha impulsado que en los últimos años se implementen políticas que fomenten el aumento de la eficiencia energética en los equipos de uso final de energía. Los sistemas accionados con motores eléctricos son los principales consumidores de la energía eléctrica, esto ha promovido el desarrollo de tecnologías para el incremento de la eficiencia de los motores eléctricos. Este trabajo muestra los resultados de un estudio de vigilancia tecnológica sobre las tendencias en las tecnologías de diseño, la normatividad y reglamentación de motores de alta eficiencia. Asimismo, se presenta el estado de la investigación e innovación en el campo de los motores eléctricos de alta eficiencia y un reporte sobre las capacidades y los actores de investigación en este campo a nivel internacional y en Colombia.

Palabras clave: Motores eléctricos de alta eficiencia; Normatividad; Políticas energéticas; Tendencias de desarrollo tecnológico; Tecnologías de diseño; Vigilancia tecnológica.

\begin{abstract}
In recent years, energy and environmental crisis has driven that implement policies that promote increased energy efficiency in end-use equipment power. The electric motors driven systems (EMDS) are the biggest consumers of electricity, this has promoted the development of new technologies to increase the efficiency of electric motors. This work shows the results of a technology watch study on trends in design technologies, standardization and regulations on energy efficient motors. The state of research and innovation in the field of energy efficient motors and a report on capability and research actors in this field in the world and Colombia is also presented.
\end{abstract}

Keywords: Energy efficient motors; standardization; Energy policies; Trends in technology development; Technology of design; Technology watch. 


\section{INTRODUCCIÓN}

El mundo enfrenta actualmente un problema energético y ambiental sin precedentes, lo cual hace necesario tomar acciones inmediatas para reducir el consumo de combustibles fósiles y las emisiones contaminantes.

Los sistemas accionados con motores eléctricos consumen aproximadamente el $46 \%$ de la energía eléctrica mundial, [1], y si consideramos que aproximadamente el $68 \%$ de la electricidad en el mundo se produce mediante combustibles fósiles [2], el aumento de la eficiencia de operación de los motores eléctricos es una de las acciones más importantes para reducir las emisiones de gases efecto invernadero. La eficiencia de operación de los sistemas accionados por motores eléctricos (EMDS) se puede incrementar tanto por gestión energética [3] - [4], como por cambios tecnológicos en el diseño del motor y por estrategias de operación óptima [5] - [6].

En la Fig. 1 se muestra la distribución de la demanda de la energía eléctrica por usuario final [1].

Figura 1. Distribución estimada de la demanda global de energía eléctrica por usuario final.

Figure 1. Estimated distribution of the global demand for electrical energy by end user.

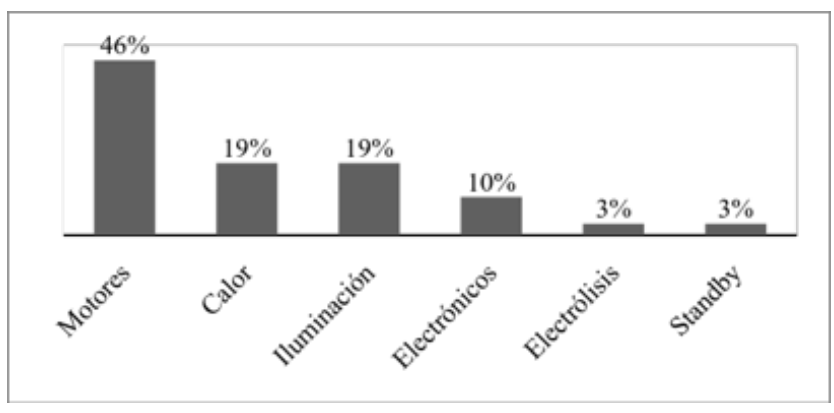

Fuente: Adaptada de Waide, P. and Brunner 2011.

En este contexto se originó una tendencia mundial, fundamentalmente en USA y Europa, de implementación de normas y regulaciones que fomentaron el desarrollo de motores de alta eficiencia [7].

Una de las acciones que impulsaron el desarrollo de los motores de alta eficiencia fueron los reglamentos que obligan a usar motores con una eficiencia mínima, éstos son conocidos como los MEPS (Minimum Energy Performance Standards) y entre ellos están el EPACT 92 (Energy Polyce Act of 1992), el EISA 2007 (Energy Independence and Security Act of 2007) y la EuP 2009 (Energy using Products directive of 2009).

Otro avance importante fue la unificación internacional de las clases de eficiencia definidas por la norma IEC y la ANSI-NEMA, estas ahora se han unificado en cinco clases: la IE1 (Eficiencia Estándar), la IE2 (Eficiencia NEMA Energy Efficient), la IE3 (Eficiencia NEMA
Premium), la IE4 (Eficiencia NEMA Super Premium) y la IE5 (Eficiencia NEMA Ultra Premium).

Respecto a las nuevas tecnologías para el incremento de la eficiencia se han desarrollado tecnologías para reducir las pérdidas del motor por medio de la eliminación del devanado del rotor, que elimina las pérdidas por efecto Joule en el devanado del rotor. Esto implica el desarrollo de nuevas tecnologías como la inclusión de imanes permanentes en el rotor ó un rotor de reluctancia variable. Entre estas tecnologías están los motores sincrónicos de imán permanente, los motores sincrónicos de imán permanente de arranque directo, los motores sincrónicos de reluctancia, los motores de reluctancia conmutada.

El presente artículo muestra los resultados parciales de un estudio de vigilancia tecnológica de gestión de motores eléctricos, que fue desarrollado por el grupo de investigación Electrical Machines \& Drives de la Universidad Nacional de Colombia Sede Bogotá, con el apoyo del Grupo de Investigación en Energías GIEN de la Universidad Autónoma de Occidente y el seguimiento del grupo de investigación GRISEC de la Universidad Nacional de Colombia Sede Bogotá. El estudio se enmarca dentro del proyecto de Gestión de Energía en Motores Eléctricos GeMe, el cual fue propuesto dentro del programa: Consolidación de la Red de conocimiento en Eficiencia Energética y su impacto en el sector productivo bajo los estándares internacionales. Con este estudio se busca efectuar un análisis sistemático y permanente con el fin de construir y actualizar la base de conocimiento en tecnologías, tendencias globales y requerimientos de mercado en el uso de los motores eléctricos y su gestión energética.

\section{METODOLOGÍA}

\subsection{Objeto de estudio}

El objetivo principal del estudio de vigilancia tecnológica fue construir y actualizar la base de conocimiento en tecnologías, tendencias globales y requerimientos de mercado en el uso de los motores eléctricos y su gestión energética, que permite específicamente identificar actores nacionales e internacionales en el tema (autores, países, instituciones, redes, grupos), establecer las dinámicas y evolución del tema, conocer la producción científica y patentes internaciones. Asimismo, ubicar a Colombia en ese contexto y recopilar la información pertinente para la toma de decisiones en la formulación de futuros proyectos de investigación, participación de convocatorias y búsqueda de financiamiento.

\subsection{Metodología usada en el estudio de vigilancia tecnológica}

Para el desarrollo de este estudio se utilizó el ciclo de vigilancia tecnológica, que fue adaptado por el grupo 
de investigación Electrical Machines \& Drives EM\&D a partir de la metodología propuesta por Sánchez-Torres y Palop-Marro [8]. El ciclo tiene básicamente las siguientes cuatro grandes etapas:

- Planeación a través de la definición de una ficha de definición de necesidades.

- Búsqueda y captación de información acerca de la temática definida.

- Análisis, validación y organización de la información recolectada.

- Inteligencia y conclusiones con base en los resultados y análisis hechos.

Finalmente se realiza el proceso de comunicación y difusión del estudio efectuado.

La metodología se inicia con la definición de necesidades de vigilancia tecnológica, estableciéndose el tema principal de la vigilancia, el objetivo general, los objetivos específicos, los factores críticos de vigilancia, las preguntas claves de vigilancia y los términos clave para las búsquedas. Así mismo la ficha describe las fuentes de búsqueda y los restrictores que sean pertinentes para la misma.

Como parte de la etapa de planeación se definieron siete factores críticos de vigilancia (FCV) y para la parte de tecnologías de diseño se definieron dos preguntas clave de vigilancia (PCV) para dar solución a dichos FCV. Las PCV fueron:

P1/ ¿Qué regulaciones, incentivos y normatividades en uso racional y eficiente de la energía se están investigando e implementando a nivel mundial?

P2/ ¿Qué tecnologías, estrategias de diseño y operación de motores eléctricos y Electric Motor Drives Systems (EMDS) se están desarrollando y utilizando a nivel mundial para lograr niveles de eficiencia: premium efficient (IE3), super-premium efficient (IE4) y Ultra-Premium efficient (IE5) en su funcionamiento como parte de sistemas de gestión integral de energía?

La solución de las PCV se hizo mediante la identificación de un estado del arte a partir de la búsqueda de publicaciones científicas, sobre la búsqueda y análisis de patentes. Para la búsqueda de publicaciones se utilizaron bases de datos científicas estructuradas como lo son Scopus y Web of Knowledge en el producto Web of Science (ISI WoS), y no estructuradas como Google Academic.

Para la captación de patentes, se buscó información en bases de datos internacionales, tales como la base de datos internacional (WIPO) y la europea (ESPACENET). Adicionalmente, se hicieron búsquedas en Google Patents con el fin de conocer las invenciones, los inventores, las entidades y las empresas que presentan las patentes y la dinámica inventiva en el tema del estudio.

La información captada se consignó en forma de corpus bibliográfico. Estos corpus obtenidos en las bases de datos estructurados fueron incorporados en programas de gestión bibliográfica para ser filtrada, clasificada y depurada (ej., eliminación de publicaciones duplicadas entre bases de datos) de acuerdo con la ficha de definición de necesidades planteada, usando las herramientas Vantage Point y Excel. De este proceso, se logró un resultado final de 185 publicaciones científicas relacionadas con las regulaciones, normas y tecnologías de diseño, las cuales se repartieron de la siguiente manera: Preguntas P1 / 41 publicaciones entre 2005-2014 y Preguntas P2 / 144 publicaciones entre 2005-2014.

A partir de este proceso se obtuvo un corpus final, el cual fue cargado a un programa especializado para el análisis y gestión de la información. En este aplicativo la información obtenida fue categorizada, procesada y analizada, generando distintas representaciones gráficas de tendencias de evolución, ranking de autores, ranking de instituciones, ranking de países, correlaciones entre palabras clave, autores, entidades, países, etc.

A partir de esta información cienciométrica se analizaron los escenarios y se formularon las conclusiones que sirven como herramientas para la toma de decisiones por parte de los sectores interesados. Adicionalmente, se organizó la información pertinente para resolver las PCV planteadas en la ficha de definición de necesidades [9].

Como aporte adicional del estudio de vigilancia tecnológica, se identificaron los actores académicos nacionales en este tema, mediante la búsqueda de grupos de investigación y entidades que han desarrollado proyectos de investigación, han publicado o en general, han trabajado en algún momento en el tema de vigilancia.

Para este fin se hicieron búsquedas en la base de datos de la plataforma ScienTI y la base de datos Publindex.

\section{TENDENCIAS EN LA REGULACIÓN Y NORMA- TIVIDAD}

Considerando que de la energía total utilizada a nivel industrial, aproximadamente el $66 \%$ la consumen los sistemas accionados por motores eléctricos y que el motor eléctrico más usado es el motor de inducción de jaula de ardilla, surgió entre las medidas más prometedoras para el ahorro de energía, establecer el incremento de la eficiencia de este tipo de motores [5]-[6].

En este contexto un estudio realizado en 1990 por el Departamento de Energía de USA mostró el potencial de ahorro que se podía obtener reemplazando motores de eficiencia estándar, por otros que fueran de 2 a $6 \%$ más eficientes. En este escenario el año 1992 se aprobó 
en USA EPACT 92, que estableció el uso obligatorio de los motores de alta eficiencia en USA a partir de 1997. Las eficiencias mínimas exigidas por el EPACT 92 fueron publicadas en la Tabla 1 de la Norma NEMA MG1.1997. Este tipo de motor se le denomino motor NEMA Energy Efficient y en español se llamó motor de alta eficiencia [6].

El EPACT 92 marcó la aparición de los MEPS (Minimum Energy Performance Standards), de hecho, el EPACT 92 fue el primer MEPS que surgió en el mundo. El MEPS es una reglamentación dada en un país que hace obligatorio la adopción de una eficiencia mínima para los motores eléctricos, con el objetivo de incrementar la eficiencia energética del país [10].

El 2007 se firmó en USA el EISA 2007, con la participación de la NEMA que estableció un nivel superior de eficiencia denominado Premium y estableció que éste sea obligatorio en USA a partir del 2015 para motores de hasta $375 \mathrm{~kW}$ y a partir de 2017 para todos los motores. Asimismo, la NEMA proyectó el motor de eficiencia Super Premium [11].

Algo similar ocurrió en la Unión Europea, pues el año 2009 se aprobó la Directiva de Ecodiseño, Energy Using Products (EuP 2009), MEPS que reglamentó el uso obligatorio de los Motores de Alta eficiencia (equivalente al EPACT 92) a partir de 2011. Además, dispuso que a partir del 2015 se debe usar motores de Eficiencia Premium entre $7.5 \mathrm{~kW}$ hasta $350 \mathrm{~kW}$ y a partir de 2017 desde $0.75 \mathrm{~kW}$ hasta $350 \mathrm{~kW}$ [12].

El 2008 la IEC emitió la Norma IEC 60034-30, [13], que estableció las clases de eficiencia IE1, IE2, IE3, IE4 y una equivalencia entre la eficiencia de los motores IEC y NEMA. Así el IE1 es el motor de Eficiencia Estándar, el IE2 es el motor de alta eficiencia ó NEMA Energy Efficient, el IE3 es el motor de eficiencia NEMA Premium y el IE4 es el motor de eficiencia NEMA Super Premium. En la versión del 2014 la Norma IEC 60034-30-1 [14], se estableció la clase IE5 equivalente la eficiencia NEMA Ultra-Premium. Véase la Tabla 1.

Tabla 1. Equivalencia en las clases de eficiencia establecidas por la Norma IEC y NEMA.

Table 1. Equivalence in the efficiency classes established by the IEC and NEMA standards.

\begin{tabular}{ccc} 
Niveles de Eficiencia & \multicolumn{2}{c}{ Clases de Eficiencia } \\
\cline { 2 - 3 } & IEC (Internacional) & NEMA (USA) \\
\hline Estandard & IE1 & - \\
\hline Alta & IE2 & Energy Efficient EPAct \\
\hline Premium & IE3 & Premium \\
\hline Super-Premium & IE4 & Super-Premium \\
\hline Ultra-Premium & IE5 & Ultra-Premium \\
\hline Fuente: Adaptada de IEC 60034-30-1 y NEMA MG1-2014.
\end{tabular}

Es importante remarcar que la clasificación de clases de eficiencia está relacionada con la disminución de pérdidas, así el motor NEMA Premium (IE3), presenta $15 \%$ menos de pérdidas respecto al motor EPAct92 (IE2). El motor NEMA Super-Premium (IE4), está diseñado para tener 15\% menos de pérdidas respecto al motor Premium (IE3). Y el motor NEMA Ultra-Premium (IE5) está proyectado para tener $20 \%$ menos de pérdidas que el motor IE4.

Es evidente que tener una equivalencia entre las clases de eficiencia de motores implica una misma forma de medirla. Hasta antes de 2007 existía una diferencia en la forma de determinar las pérdidas adicionales, mientras la IEEE 112 B usaba un procedimiento experimental para evaluarla, la IEC 60034-2 estimaba estas pérdidas como un porcentaje de la potencia nominal, este hecho daba diferencias en la evaluación de la eficiencia [15]. Sin embargo, el año 2007 se unificó la forma de medir la eficiencia, pues se expidió la norma IEC 60034-2-1 que presentó un método equivalente al IEEE 112 B. Asimismo en su versión del 2014 la IEC 60034-2-1 [16] establece una equivalencia más ajustada con los métodos de la Norma IEEE 112 [17].

En este contexto es importante mencionar que la norma IEC 60034-2-3 se puede utilizar para determinar la eficiencia del conjunto motor de inducción y variador de frecuencia [18]. Asimismo, que la Norma IEC 60034-30-2, incluye los niveles de eficiencia de motores que operan con variadores de frecuencia, y en este caso la eficiencia tiene que ser considerada con las variaciones de la velocidad y el par [19].

En septiembre de 2015 en Colombia, el Ministerio de Minas y Energía emitió la Resolución 41012 para expedir el Reglamento Técnico de Etiquetado RETIQ con fines de uso racional de la energía, aplicable a equipos de uso final de energía eléctrica y gas combustible, para su comercialización y uso en Colombia. El RETIQ constituye el primer MEPS en Colombia. La Resolución dispuso la entrada en vigencia del RETIQ a partir del 31 de agosto de 2016. Asimismo, el Anexo General del RETIQ establece las eficiencias mínimas de comercialización para motores trifásicos de inducción de jaula de ardilla, de hasta $600 \mathrm{~V}, 60 \mathrm{~Hz}$, con potencias desde $0,18 \mathrm{~kW}$ hasta $373 \mathrm{~kW}$, abiertos o cerrados. Al entrar en vigencia el RETIQ se prohibió comercializar motores trifásicos con eficiencia menor a 50\%, a partir del 31 de agosto de 2017 solo se podrá comercializar motores de clase de eficiencia IE1 y superiores, dos (2) años después el límite mínimo corresponderá con el límite inferior de la clase de eficiencia IE2, cuatro (4) años después el límite mínimo corresponderá con el límite inferior de la clase de eficiencia IE3 para motores con potencias iguales o superiores a $7,5 \mathrm{~kW}$ y a los cinco (5) años de entrada en vigencia del RETIQ el límite mínimo corresponderá con el lí- 
mite inferior de la clase de eficiencia IE3 para motores con potencias iguales o superiores a $0.75 \mathrm{~kW}$ [20].

\section{TENDENCIAS EN EL DESARROLLO DE TEC- NOLOGÍAS DE DISEÑO}

En el objetivo de aumentar la eficiencia de los motores eléctricos, existen varias tendencias para disminuir las pérdidas en el motor mediante la aplicación de nuevas tecnologías.

La tecnología de los motores de inducción de jaula de ardilla (SCIM Squirrel-Cage Induction Motor) es la tecnología más madura. Los SCIM son los motores más usados actualmente, y fueron los primeros motores de alta eficiencia EPACT 92 (IE2) que se desarrollaron. Esta tecnología ha sido usada para desarrollar el motor de eficiencia IE3 y también el IE4. Una gran cantidad de publicaciones presentan resultados en técnicas de diseño para el mejoramiento de la eficiencia en estos motores, tales como el alargamiento del núcleo de la máquina, la inyección de la jaula de ardilla en cobre, el recocido de la lámina del rotor entre otros [21, 22]. Estas estrategias se conocen como NTC (No-Tooling Cost), ya que son pensadas de tal forma que no se requiere de un elevado costo en inversión de nuevo herramental para su fabricación. Sin embargo, la tecnología SCIM tiene limitaciones para cumplir con la clase de eficiencia IE4, y no permite implementar el nivel de eficiencia IE5.

Una de las vías para reducir las pérdidas del motor, se ha encontrado eliminando el devanado del rotor, lo que elimina las pérdidas por efecto Joule en el devanado del rotor, incrementando así la eficiencia. Esto implica el desarrollo de nuevas tecnologías como la inclusión de imanes permanentes en el rotor ó cambiar el concepto de producción de par electromagnético usando el par de reluctancia. Estas tecnologías permiten cumplir con la clase de eficiencia IE4 [7].

Para aplicaciones de velocidad fija se desarrolló el motor de imanes permanentes de arranque en línea (Line-Start Permanent Magnet Synchronous Motors - LS$P M S M)$. Este motor lleva en el rotor imanes permanentes y una jaula de ardilla, que le permite arrancar directamente con la tensión de la red y luego al rotor entrar en sincronismo con el campo del estator. Aunque solo puede operar a la velocidad sincrónica, tiene la ventaja que arranca con la tensión de línea y puede intercambiarse directamente con un motor tipo SCIM, pues llevan el mismo tamaño de carcasa.

Para aplicaciones de velocidad variable están los motores sincrónicos de imán permanente (PMSM Permanent Magnet Synchronous Motor), los motores sincrónicos de reluctancia (SynRM Synchronous Reluctance Motor), los motores de reluctancia conmutada (SRM Switched Reluctance Motor).
Los PMSM llevan un devanado similar al motor de inducción, pero llevan imanes en el rotor. Estos pueden ponerse en servicio mediante un variador de frecuencia. En este tipo de motores se estudia la aplicación de nuevos materiales magnéticos enrarecidos para la construcción de los imanes permanentes.

El SynRM es un motor que tiene un devanado de estator similar al SCIM, pero un diseño especial del núcleo del rotor que hace que el campo magnético del estator se enclave con el rotor, al ofrecer una reluctancia mínima al campo magnético [23]. Se ha identificado últimamente un aumento en el número de investigaciones relacionadas con el diseño de motores SynRM asistidos por imanes permanentes [9].

Los $S R M$, aplican un diseño con saliencias en el estator y el rotor, y no lleva devanado en el rotor [24]. En los SRM el par se forma por el principio de reluctancia mínima, asimismo para ponerse en operación, el motor necesita ser alimentado con un controlador de potencia para alimentar secuencialmente las bobinas ubicados en las saliencias del núcleo del estator.

Finalmente, aunque la tecnología para lograr eficiencias IE5 aún está en investigación, se plantea la idea de migrar de tecnologías que basan su funcionamiento en flujos radiales de campos magnéticos a aquella que aplican diseños con flujos axiales, tales como los motores sincrónicos de imanes permanentes con flujo axial [25-28].

\section{ACTORES Y CAPACIDADES}

La tendencia del desarrollo de nuevas tecnologías de diseño de motores de alta eficiencia muestra un incremento constante en el número de patentes, así el año 2014 se tuvo el pico en patentes, teniéndose en promedio 6 patentes por año. Los países líderes que tienen el mayor número de patentes en este mismo periodo son USA, China, Japón y Corea del Sur, esta tendencia concuerda con los países que son líderes en el número de publicaciones.

El mayor número de patentes se encontró en el diseño de motores de imanes permanentes, sobre todo en el diseño de motores de imanes permanentes con arranque en línea, una propuesta en esta tendencia es el uso de sistemas de multi-conexión con el fin de ajustar el flujo magnético en función de la carga del motor [7]. Le siguen los motores de reluctancia, siendo la tecnología SynRM el que tiene la mayor tendencia. En menor medida se encontraron patentes en la tecnología SCIM, donde la tendencia se alinea con las estrategias NTC (No-Tooling Cost), que aplica medidas tales como el alargamiento del núcleo de la máquina, la inyección de la jaula de ardilla en cobre, el recocido de la lámina del rotor. También se evidencia 
patentes en diseños para automóviles eléctricos y para la industria automovilística en general, sobre todo de motores de reluctancia y de imán permanente.

En el campo de las normatividades, regulaciones y metodologías para el uso eficiente de energía en aplicaciones industriales, entre el 2004 y el 2014, se encontraron 41 artículos científicos. Los países líderes en publicaciones en este tema están USA, seguido Malasia y Portugal, y a nivel latinoamericano están Brasil y Colombia. Finalmente, a nivel de autores se destacan el Profesor T. M. Indra Mahlia de la Universiti Tenaga Nasional y el Profesor A. T. de Almeida de la Universidad de Coimbra.

En relación a las publicaciones realizadas entre los años 2004 y 2015 por actores nacionales en el tema de eficiencia energética, diseño, monitoreo y diagnóstico en motores eléctricos y EMDS, se identificó que las ciudades de Bogotá, Cali, Bucaramanga y Medellín lideran el número de publicaciones en el tema. Asimismo, se encuentra que las universidades que lideran la lista de publicaciones en este campo son: la Universidad Nacional de Colombia, la Universidad del Valle y la Universidad Autónoma de Occidente.

Los países de América Latina, a excepción de Brasil y México, cuentan con un número limitado de laboratorios acreditados para estos procedimientos. Según la base de datos de la Red Nacional de Laboratorios, la ONAC y el SIC en Colombia no existen a la fecha laboratorios acreditados para pruebas en motores eléctricos [29]. Sin embargo, se identificaron 12 universidades y una empresa industrial con un potencial básico para la implementación de pruebas para la determinación de eficiencia en motores eléctricos y conjuntos Drive-Motor-Carga [9]. Considerando que la Resolución 41012 de septiembre de 2015 estableció que el Reglamento Técnico de Etiquetado RETIQ es de obligatorio cumplimiento a partir del 31 de Agosto de 2016, se hace evidente que el desarrollo de laboratorios acreditados para el ensayo de eficiencia de motores eléctricos es una acción urgente en Colombia, pues son los organismos que tendrán que certificar la eficiencia que aparecerá en la etiqueta de datos que deberán llevar los motores eléctricos en cumplimiento de este reglamento.

\section{RESULTADOS Y DISCUSIÓN}

A nivel internacional se ha logrado la unificación en la clasificación de niveles de eficiencia de motores eléctricos y del método aplicado para su determinación. Así, la Norma IEC 60034 30-1 y la Norma ANSI NEMA MG1 definen cinco clases de eficiencia: IE1, IE2, IE3, IE4 e IE5. Además, la Norma IEC 60034 2-1 y la Norma IEEE 112 aplican métodos equivalentes para la determinación de la eficiencia de motores. En Co- lombia, legalmente se reconoce la norma IEC, pues, el organismo de normalización Icontec tiene el comité 131 que es un comité espejo del comité técnico de motores de la IEC.

Existe una tendencia mundial de establecer reglamentos de cumplimiento obligatorio que designan la eficiencia mínima para la comercialización de motores eléctricos, éstos son conocidos como MEPS (Mínimum Energy Performance Standard). Los MEPS son elementos muy importantes para el incrementar la utilización de motores de alta eficiencia. El primer MEPS de motores eléctricos se emitió en USA en 1992 (EPACT 92), posteriormente se estableció en la EU y Japón.

Los MEPS han reglamentado el uso del motor de eficiencia clase IE3 a partir del 2015 en USA y en la EU. En Colombia el RETIQ es el primer MEPS en motores eléctricos y entró en vigencia el 29 agosto 2016. A nivel de Latinoamérica, México, Brasil y Colombia, son los únicos países que han emitido MEPS.

Para la construcción de motores de clase de eficiencia IE3, la tecnología más investigada es la de motores tipo jaula de ardilla. Sin embargo, para las clases IE4 y IE5 se está investigando nuevas tecnologías tales como rotor de Imanes Permanentes y de Reluctancia.

Para la clase IE5 la tecnología SCIM está descartada y se abre un tema de investigación para nuevas tecnologías y diseños de motores para lograr eficiencias Ultra-Premium/IE5. Las tecnologías identificadas para los motores de alta eficiencia son: motores de imanes permanentes (se investiga la aplicación de nuevos materiales magnéticos enrarecidos), los motores de reluctancia (se investiga la aplicación de motores de reluctancia variable), motores de imán permanente con arranque directo en línea, de motores de sincrónicos de reluctancia y motores de reluctancia asistidos con imanes permanentes. El mayor porcentaje de patentes se está registrando en el diseño de motores de imanes permanentes, donde la tecnología LSPMSM presenta una tendencia ascendente. Luego están los motores de reluctancia, donde se encontraron patentes especialmente de diseños en motores sincrónicos de reluctancia. En las máquinas de inducción tipo jaula de ardilla, el número de patentes fue menor.

A nivel internacional los países que tienen el mayor número de patentes y publicaciones son USA, China, Japón y Corea del Sur, seguidos de países europeos como Alemania, Italia y Portugal. A nivel latinoamericano se encuentran Brasil, Argentina, Colombia y México. Respecto a las instituciones, la Universidad Nacional de Colombia es la líder en publicaciones en el tema de investigación de motores eléctricos, seguida por la Universidad del Valle, la Universidad Autónoma de Occidente, la Universidad Industrial de 
Santander y la Universidad Distrital Francisco José de Caldas.

Respecto a laboratorios acreditados para la ejecución de ensayos de eficiencia a motores eléctricos, en Colombia no existen este tipo de laboratorios. Sin embargo, la investigación encontró que el país cuenta con 12 universidades con laboratorios de máquinas eléctricas con potencial de ser acreditados para esta prueba, y un laboratorio a nivel industrial. Por lo tanto, es urgente impulsar la acreditación de laboratorios en Colombia sobre todo considerando que el Reglamento de Etiquetado de equipos de uso final RETIQ entró en vigencia desde agosto de 2016.

\section{CONCLUSIONES}

El estudio de vigilancia tecnológica en motores eléctricos logró construir una base de conocimiento sobre las tecnologías, las tendencias globales y los requerimientos del mercado en el uso de motores eléctricos.

Actualmente se ha logrado la unificación para clasificar y determinar la eficiencia de los motores eléctri$\cos$ a nivel mundial. Así existe una equivalencia en las normas de USA (NEMA, IEEE) y la EU (IEC).

USA fue el primer país del mundo en emitir el MEPS para motores eléctricos, luego le siguió la EU. A nivel latinoamericano el primer país fue México, seguido por Brasil y Colombia. Los MEPS han demostrado ser una herramienta fundamental para el incremento del uso de motores de alta eficiencia.

Se han desarrollado nuevas tecnologías para Construir motores eléctricos de clase de eficiencia IE4 e IE5, tales como una tecnología de motores de imanes permanentes y motores de reluctancia. La tecnología de motores de jaula de ardilla está descartada para motores de clase de eficiencia IE5.

A nivel mundial USA lidera la producción de patentes y publicaciones en el campo de motores eléctricos de alta eficiencia, a nivel latinoamericano es líder Brasil.

En Colombia no existen laboratorios acreditados para el ensayo de eficiencia de motores eléctricos, sin embargo, existen aproximadamente 13 instituciones que tienen laboratorios con potencial de ser acreditados. Con la entrada en vigencia del RETIQ (Agosto 2016) es importante que Colombia tenga laboratorios acreditados para determinación de eficiencia de motores eléctricos.

\section{AGRADECIMIENTOS}

Los autores expresan su agradecimiento a Colciencias, por la financiación del proyecto "Gestión de Energía en Motores Eléctricos en Sistemas Eléctricos Industriales - GeMe". Proyecto desarrollado en el marco de la Red Colombiana de Conocimiento en Eficiencia Energética RECIEE, del cual el presente artículo es un producto parcial.

\section{REFERENCIAS}

[1] P. Waide \& C. U. Brunner, Energy-efficiency police opportunities for electric motor-driven systems, Paris, Francia, International Energy Agency, 2011, pp. 29-41.

[2] International Energy Agency. (2017) Key World Energy Statistics, [Internet], France, International Energy Agency. Disponible desde: <http://www.iea.org/statistics> [Acceso 20 enero 2017].

[3] R. del P. Castrillón-Mendoza, A. J. González-Hinestroza, \& E. C. Quispe, "Mejoramiento de la eficiencia energética en la industria del cemento por proceso húmedo a través de la implementación del sistema de gestión integral de la energía", DYNA, vol. 80, (177), 115-123, 2013.

[4] R. del P. Castrillón-Mendoza, E. C. Quispe, A. J. E. GonzálezHinestroza, M. Urhan \& D. Fandiño-Valencia, Metodología para la implementación del Sistema de Gestión Integral de la Energía. Fundamentos y casos prácticos. Colombia: Editorial Universidad Autónoma de Occidente, 2014, pp. 13-29.

[5] E. C. Quispe, "Una Visión Integral para el Uso Racional de la Energía en la Aplicación de los Motores Eléctricos de Inducción", El Hombre y la Máquina, vol. XV, (20-21), 52-59, 2003.

[6] E. C. Quispe \& L. F. Mantilla, "Motores eléctricos de alta eficiencia: características electromecánicas, ventajas y aplicabilidad", Energía y Computación, vol. XII, (1), 11-19, 2004.

[7] A. T. De Almeida, F. J. T. E. Ferreira \& G. Baoming, "Beyond induction motors - Technology trends to move up efficiency", IEEE Transactions on Industry Applications, vol. 50, (3), 21032114, 2014.

[8] J. M. Sánchez-Torres, Herramientas de software para la práctica en la empresa de la vigilancia tecnológica e inteligencia competitiva. España: Triz XXI, 2002, pp. 19-38.

[9] J. Rosero \& S. F. Contreras, (2015) Informe de Vigilancia Tecnológica. Proyecto Gestión de la Energía en Equipos de Uso Final: Motores Eléctricos - Contrato 0464 de 2013, Universidad Nacional de Colombia.

[10] E. B Agamloh, N. Kaufman \& K. Butler. (2007) The Economics of Pre-EPAct Motors Operating in Industry. Conference Record of Annual IEEE Pulp and Paper Industry Technical Conference. 2013.

[11] E. C. Quispe, (2015), Jun./Jul., Recomendaciones para la aplicación de motores de alta eficiencia, Reportero Industrial [on line], 83 (3), p. 16-18. Disponible desde: < http:/ / www. reporteroindustrial.com> [Acceso 20 de enero 2018].

[12] A. T. De Almeida, F.J. T. E. Ferreira, J. A. C. Fong \& Brunner (2008) Electric motor standards, ecodesign and global market transformation. IEEE Industrial and Commercial Power Systems Technical Conference. 2008. 
[13] “Rotating Electrical Machines - Part 30: Efficiency classes of line operated AC motors (IE code), IEC 60034-30 Standard", 2008.

[14] "Rotating Electrical Machines - Part 30-1: Efficiency classes of line operated AC motors (IE code), IEC 60034-30-1 Standard", 2014.

[15] A. T. De Almeida, F. J. T. E Ferreira, J. F. Busch \& P. Angers, "Comparative analysis of IEEE 112-B and IEC 34-2 efficiency testing standards using stray load losses in low-voltage threephase cage induction motors", IEEE Transactions on Industry Applications, vol. 38, (2), 608-614, 2002.

[16] "Rotating Electrical Machines - Part 2-1: Standard methods for determining losses and efficiency from tests, IEC 60034-2-1 Standard", 2014.

[17] “IEEE Standard 112: IEEE Standard Test Procedure for Polyphase Induction Motors and Generators, IEEE Std $112^{\mathrm{TM}}$ $-2004^{\prime \prime}, 2004$.

[18] "Rotating Electrical Machines - Part 2-3: Specific test methods for determining losses and efficiency of converter-fed AC induction motors, IEC 60034-2-3 Standard", 2013.

[19] "Rotating Electrical Machines - Part 30-2: Efficiency classes of variable speed AC motors (IE code), IEC 60034-30-2 Standard", 2016.

[20] "Resolución 41012 del 18 de septiembre de 2015 - Ministerio de Minas y Energía de Colombia: Reglamento Técnico de Etiquetado, RETIQ", 2015.

[21] I. Peter, G. Scutaru \& C. G. Nistor. (2014) Manufacturing of asynchronous motors with squirrel cage rotor, included in the premium efficiency category IE3, at S.C. Electroprecizia Electrical-Motors S.R.L. Sacele. International Conference on Optimization of Electrical and Electronic Equipment, OPTIM 2014. 2014.

[22] L. Alberti, N. Bianchi, A. Boglietti \& A. Cavagnino, “Core axial lengthening as effective solution to improve the induction motor efficiency classes", IEEE Transactions on Industry Applications, vol. 50, (1), 218-225, 2014.

[23] P. R. Viego, J. R. Gómez \& E. C. Quispe, “Motores sincrónicos de reluctancia controlados con variadores de frecuencia: una aplicación para ahorrar energía", Ingeniería Energética, vol. 36, (1), 72-22, 2015.

[24] S. M. Castano \& J. Maixé-Altés, “Design and sizing of a switched reluctance motor for an automotive application", DYNA, vol 80, (181), 81-90, 2013.

[25] A. T. De Almeida, F. J. T. E. Ferreira \& A. Q. Duarte, “Technical and economical considerations on super high-efficiency threephase motors", IEEE Transactions on Industry Applications, vol. 50, (2), 1274-1285, 2014.

[26] D. G. Dorrell, "A Review of the Methods for Improving the Efficiency of Drive Motors to Meet IE4 Efficiency Standards", Journal of Power Electronics, vol. 14, no. 5, pp. 842-851, Sept. 2014.

[27] X. Feng, Y. Bao, L. Liu, L. Huang \& Y. Zhang. (2012, Sept.) Performance investigation and comparison of Line Start-up Permanent Magnet Synchronous Motor with super premium efficiency. 20th International Conference on Electrical Machines - ICEM 2012. 2012.
[28] F. J. T. E Ferreira, G. Baoming \& A. T. De Almeida. (2012, Sept.) Stator winding connection mode management in linestart permanent magnet motors to improve their efficiency and power factor. 20th International Conference on Electrical Machines - ICEM 2012. 2012.

[29] C. M. Londoño \& J. L. Ramírez, "Normas de Eficiencia Energética de Motores de Inducción, ¿Está Preparada Latinoamérica?", Tecno Lógicas, (30), 117-147, 2013. 